\title{
LAS NORMAS INTERNACIONALES DE INFORMACIÓN FINANCIERA: HISTORIA, IMPACTO Y NUEVOS RETOS DE LA IASB
}

\author{
Nadia Ugalde Binda ${ }^{1}$ \\ Recibido: 12/11/2013 \\ Aprobado: 05/03/2014
}

\begin{abstract}
RESUMEN
La internacionalización de las transacciones comerciales de la postguerra, la expansión empresarial a través de fusiones y adquisiciones así como el interés de abarcar carteras de inversiones más diversificadas, obligan a los organismos a redactar estándares contables uniformes en todos los países que permitan la comparabilidad de la información financiera para estas nuevas "compañías multinacionales" (Zeff, 2012). Así surge en 1973 la IASC, con sede en Londres como un primer intento de establecer los estándares básicos internacionales llamados Normas Internacionales de Contabilidad. En este artículo se hace un recorrido histórico de las instituciones involucradas en la formulación de estas normas, las implicaciones que su aplicación ha tenido sobre los estados financieros, cómo algunos países han hecho adaptaciones a las mismas y cuáles son los principales retos que la IASB debe afrontar en el futuro.
\end{abstract}

PALABRAS CLAVES: NORMAS, CONTABILIDAD, COMPARABILIDAD, INFORMES FINANCIEROS.

\begin{abstract}
The internationalization of business transactions, business expansion through mergers and acquisitions, and the interest of covering more diversified investment portfolios, require agencies to draft uniform accounting standards across countries to allow comparability of financial information for these new "multinational companies" (Zeff, 2012). Thus, in 1973 arises the IASC, based in London as a first attempt to establish international basic standards called International Accounting Standards. This article is a historical overview of the institutions involved in the formulation of these rules, the implications that its implementation has had on the financial statements, how some countries have made adjustments to them and what are the main challenges that the IASB should face in the future.
\end{abstract}

KEY WORDS: STANDARDS, ACCOUNTING, COMPARABILITY, FINANCIAL REPORTS.

$1 \quad$ Universidad de Costa Rica, Escuela de Administración de Negocios. Código Postal 11501-2060, Costa Rica; nubaf@ hotmail.com. 


\section{INTRODUCCIÓN}

Este artículo recoge algunas inquietudes sobre la forma en que se preparan los estados financieros en diferentes países y cómo esta presentación contable puede repercutir en la toma de decisiones si no se hace sobre estándares reconocidos. Así mismo, se explica cómo existe una necesidad de presentar información comparable entre países, $y$ la evolución de esta necesidad hasta la creación de organizaciones mundiales que buscan establecer normas internacionales de contabilidad. El objetivo de estas organizaciones es que las normas contables sean aplicadas en cualquier empresa $y$ así sus estados financieros sean comparables y su información transparente sirva para ser interpretados de manera apropiada en la toma de decisiones. Finalmente, este estudio recoge algunos de los retos que las organizaciones mundiales deben salvar para lograr este objetivo de unificar las bases de presentación de la información contable al generar estándares mínimos de calidad.

\section{Antecedentes}

La incomparabilidad de la información financiera afecta las decisiones de negocios en muchas maneras. Las decisiones sobre adquisiciones y fusiones, sobre crédito, sobre inversión en el mercado global o de utilizar proveedores fuera de las fronteras; por ejemplo, pueden variar dependiendo de los estándares utilizados para preparar la información financiera relacionada. (Stanko et al. 2010).

Los países han usados sus propios estándares contables, siguiendo a líderes del comercio mundial como Estados Unidos y Europa, pero haciendo las modificaciones necesarias para adaptarlos a su entorno económico. Las políticas de los informes contables $y$ financieros varían entre países dependiendo de su nivel de desarrollo industrial, pues los países desarrollados han requerido un grado mayor de sofisticación en sus sistemas contables que las economías emergentes o países en desarrollo. Otra variable con una influencia significativa en los estándares contables es la estructura de impuestos de los países, pues en algunos se exige la determinación del ingreso gravable en concordancia con estas normas, pero en otros las autoridades gubernamentales requieren reportar los mismos ingresos gravables para efectos tributarios que en los libros e informes contables (como en Alemania, Japón y Suecia). Finalmente, los países que reciben inversión extranjera tienden a obedecer las demandas de sus proveedores de capital en cuanto a la preparación de los informes contables (Stanko et al. 2010).

La internacionalización de las transacciones comerciales de la postguerra, la expansión empresarial a través de fusiones y adquisiciones así como el interés de abarcar carteras de inversiones más diversificadas, obligan a los organismos a redactar estándares contables uniformes que permitan la comparabilidad de la información financiera para las nuevas "compañías multinacionales" (Zeff, 2012).

Un ejemplo de esta internacionalización es que, para 1997, en los mercados de capitales de Estados Unidos (país que acumula el 42\% de las negociaciones mundiales de acciones), los extranjeros registrados aportaban $\$ 28$ billones y 1.000 de las 13.000 compañías inscritas en la SEC eran extranjeras (Canfield, 1999).

\section{La creación del IASC}

Estas presiones económicas hacen que en 1973 se organice la IASC con sede en Londres, bajo el acuerdo de los representantes de profesionales contables de varios países (Alemania, Australia, Estados Unidos, Francia, Holanda, Irlanda, Japón, México y Reino Unido) para comparar las normativas existentes y tratar de disminuir las diferencias en las prácticas contables entre países. Este fue el primer intento de establecer los estándares básicos internacionales llamados Normas Internacionales de Contabilidad (Zeff, 2012).

Según Richardson y Eberlein (2011) los estándares producidos por IASC tenían la intención de ser una base de armonización de las prácticas alrededor del mundo y también una guía para los países en desarrollo, cuya falta de capacidad técnica les impidiera desarro- 
llar sus propios estándares contables. Su objetivo era generar un nivel mínimo de calidad en la información financiera.

Entre 1973 y 1987, el IASC emitió 28 normas y en 1980 gran cantidad de empresas le dieron su apoyo al reportar en su información financiera que se prepararon de forma consistente con estas normas, entre ellas General Electric, Exxon, FMC Corporation y Sasebo Heavy Industries Co. Entre los años 1987 al 2000, más empresas se adhieren a las NIC's para sus informes anuales como Nestlé, Roche, Bayer y Microsoft (Zeff, 2012).

Sin embargo, surgen dudas sobre la legitimidad de IASC para dictar normas internacionales. Se detallan deficiencias sobre todo con respecto a que una gran mayoría de usuarios de los estados financieros no estaba representado en los comités, el proceso no era transparente $y$ el uso de resultados obtenidos por el "debido proceso" en la emisión de los estándares no era evidente (Richardson y Eberlein, B., 2011). Además, los estándares emitidos al inicio compilan una serie de prácticas contables y con frecuencia permiten tratamientos alternativos para una transacción (Canfield, 1999), lo que hace que su aplicación produzca resultados múltiples, según el criterio de quien lo aplica.

\section{El cambio hacia IASB}

En mayo del 2000, los 143 profesionales provenientes de 104 países que conformaban IASC aprueban una reestructuración y una nueva constitución, cambiando su nombre a Junta de Normas Internacionales de Contabilidad, en la que se incluyeron miembros de organismos regulatorios, organizaciones internacionales, corporaciones globales (tales como Citigroup) y de la profesión contable (como KPMG y Deloitte). El IASC fue renombrado entonces como IASB y su estructura fue diseñada de manera similar a la FASB con 19 miembros, todos con experiencia como auditores, contadores, usuarios de los estados financieros y académicos, en su mayoría dedicados a tiempo completo al proyecto.

IASB es un organismo independiente del sector privado que desarrolla y aprueba las
Normas Internacionales de Información Financiera (NIIF o IFRS por sus siglas en inglés). El IASB funciona bajo la supervisión de la Fundación del Comité de Normas Internacionales de Contabilidad (IASCF). La Fundación es una institución independiente, sin fines de lucro creada en el año 2000 para supervisar al IASB. Richardson y Eberlein (2011) sugieren que las NIIFs buscan simplificar los flujos de capitales internacionales entre las economías con mercados desarrollados y están enfocados principalmente en empresas públicas grandes, multinacionales, o que cotizan en bolsa.

Desde el 2001, la emisión de normas está a cargo de la IASB. La página de IASB da acceso a documentación sobre las normas que probablemente excede lo que otros emisores de normas locales publican. Antes de la publicación oficial de una norma, se abre un periodo de consultas de 120 días que permite la apertura y toma de decisiones basada en evidencia. Además, un comité de interpretaciones de la información financiera internacional (IFRIC por sus siglas en inglés) desarrolla y solicita comentarios sobre la aplicación de las normas promulgadas por IASB (Stanko, et al. 2010).

La Junta de Normas Internacionales de Contabilidad tiene por objetivos ${ }^{2}$ :

a) desarrollar, buscando el interés público, un único conjunto de normas contables de carácter global que sean de alta calidad, comprensibles y de cumplimiento obligado, que requieran información de alta calidad, transparente y comparable en los estados financieros y en otros tipos de información financiera, para ayudar a los participantes en los mercados de capitales de todo el mundo, $y$ a otros usuarios, a tomar decisiones económicas;

b) promover el uso y la aplicación rigurosa de tales normas;

c) cumplir con los objetivos asociados con (a) y (b), teniendo en cuenta, cuando sea necesario, las necesidades especiales de entidades pequeñas $y$ medianas $y$ de economías emergentes; $y$

2 http://www.nicniif.org/home/iasb/que-es-el-iasb. html\#ObjetivosIASB 
d) llevar a la convergencia entre las normas contables nacionales y las Normas Internacionales de Contabilidad $y$ las Normas Internacionales de Información Financiera, hacia soluciones de alta calidad.

Desde su creación, IASC (de 1973 al 2000) logró emitir 41 normas que para el 2001 ya tenían su traducción oficial en alemán, francés, ruso, polaco, rumano, español y chino aunque se emiten inicialmente en inglés (Larson y Lee, 2001). Por su parte, IASB (desde el 2001) ha emitido 13 normas más (NIIF's). La labor de ambas instituciones ha reestructurado completamente la forma de reportar la información financiera logrando el apoyo de los comités nacionales, instituciones gubernamentales y reguladores de los mercados de capitales alrededor del mundo (Zeff, 2012). Así, la Unión Europea y Australia promueven la adopción de las normas nacidas de estas instituciones para el 1 de enero de 2005, cambiando para siempre el panorama contable de antes de la Segunda Guerra Mundial, cuando cada país creaba sus propios principios contables generalmente aceptados, una diversidad de métodos y prácticas contables que hacía muy difícil la comparabilidad de la información financiera entre compañías de diferentes países.

Para el 2005 efectivamente la mayoría de las 8.000 empresas inscritas en las bolsas de valores de la Unión Europea presentaron sus estados consolidados bajo las NIC's. Más tarde se unen países como Suráfrica, Nueva Zelanda, Israel, Brasil, Chile, Canadá y Corea. Para el 2010, Japón permite que sus empresas con operaciones internacionales usen las normas internacionales. Más tarde, el Banco Mundial persuade a más de 80 economías en desarrollo o emergentes a adoptar las NIIF's y NIC's (Zeff, 2012). Desde el 2007, la SEC autoriza el uso de NIIF's para los declarantes extranjeros sin el requerimiento de la reconciliación con U.S. GAAP. Otro organismo que recomendó a sus miembros permitir a las compañías multinacionales el uso de las normas de IASC fue IOSCO, una institución con sede en Montreal compuesta por más de 100 comisiones de valores y agencias gubernamentales similares (Larson y Lee, 2001).

Iniciativas particulares se dan alrededor del mundo. Pr ejemplo, España inicia el proceso de reforma y adaptación de la materia contable a las regulaciones internacionales en 2007 y aprueba el Plan General de Contabilidad, el Plan General de Contabilidad de Pequeñas y Medianas Empresas y criterios para las microempresas que rigen desde el 2008 (Fitó et al. 2010).

\section{$2 \quad$ NIIFs para Pymes}

En 2009, IASB emite las normas contables para pequeñas $y$ medianas empresas (PYMES) con el fin de simplificar su uso en ese sector empresarial específico (Zeff, 2012). Son aproximadamente 257 páginas donde se han simplificado muchos de los principios de las NIIF COMPLETAS en aspectos como el reconocimiento y la cuantificación de Activos, Pasivos, Ingresos, Costos y Gastos y revelaciones requeridas. La NIIF para PYMES no considera los siguientes temas por no ser relevantes para este tipo de empresas (Chasipanta, 2012):

\section{a. Ganancias por acción \\ b. Información financiera intermedia \\ c. Presentación de información sobre seg- mentos \\ d. Seguros (porque las entidades que ven- den al público contratos de seguro gene- ralmente se clasifican como responsables públicamente) \\ e. Activos no corrientes mantenidos para la venta (no obstante la tenencia de un activo para la venta pero si su potencial indicador de deterioro)}

La NIIF para PYMES debe ser usada por las entidades que el IASB define como sigue (Chasipanta, 2012):

a. No tienen obligación pública de rendir cuentas $y$;

b. Publican estados financieros con propósitos de información general para usuarios externos; estos usuarios son: 
los propietarios que no están implicados en la gestión del negocio, los acreedores actuales o potenciales $y$ las agencias de calificación crediticia.

Las empresas que coticen en la bolsa de valores no lo deben aplicar las NIIF para PYME, sin importar el tamaño.

\section{Impacto de las Normas Internacionales de Información Financiera}

$\mathrm{Al}$ aplicarse las NIIFs por primera vez en las empresas se han notado incrementos en el rubro de efectivo y sus equivalentes, las obligaciones de corto y largo plazo, propiedad, planta y equipo, arrendamientos financieros, así como una reducción de las provisiones $y$ algunas modificaciones en el reconocimiento del ingreso, entre otras.

En el caso de Italia, por ejemplo, hay una diferencia cercana al $14 \%$ en el beneficio neto medido según NIIFs en comparación a las normas nacionales donde se denota un impacto positivo de las normas relacionadas con combinaciones de negocios $y$ activos intangibles $y$ un impacto negativo en el tratamiento contable de las provisiones y los instrumentos financieros (Cordazzo, 2008).

En Chile, las mayores diferencias surgen de la revaluación de los activos, al aplicar la Nic 16 (Jara \& Contreras, 2010) y en Alemania, Hung y Subramanyam (2004) encuentran una variación en el total de activos y el valor en libros del patrimonio, así como en el ingreso neto que son significativamente más altos bajo las NIIFs que bajo las normas locales (HGB).

Algunas de las causas pueden ser la eliminación de la amortización de la plusvalía, la medición de los instrumentos financieros al valor de mercado, el reconocimiento de algunos costos de desarrollo y el reconocimiento de impuestos diferidos.

Para comprobar el impacto que han tenido las normas y su aplicación en la labor contable, hay varios estudios que analizan si se ha mejorado la calidad de la información financiera $y$ con ello la transparencia. En una encuesta llevada a cabo entre los socios de las grandes empresas de contabilidad en cada uno de 53 países sobre 62 medidas contables durante el 2000 , se logró determinar cuatro categorías principales de diferencias en la aplicación de las normas (Wilhelm \& Wilhelm, 2010):

1. Reglas contables nacionales vagas o generales que no proveen guías en áreas requeridas por las NIIFs por la ausencia de reglas específicas

2. Inconsistencias en las partidas principales en comparación con las NIIFs que pueden dar lugar a diferencias en muchas empresas

3. Las inconsistencias en los estándares nacionales con las NIIFs que podrían dar lugar a diferencias en ciertas empresas porque limitan el juicio y la flexibilidad.

La adaptación de normas por parte de los diferentes países, (que si bien es cierto las adoptan, lo hacen con algunas excepciones), dificulta la comparabilidad de la información, aunque parezcan diferencias muy sutiles.

Seguidamente se presentan algunos ejemplos de países, algunos suscritos a las NIIFs y otros con sus propias normas nacionales, que muestran diferencias en las revelaciones, valuaciones o presentación de la información financiera.

\section{Arabia Saudita}

Desde 1997 aplica el grupo completo de NICs, sin embargo para las inversiones sin influencia significativa se utiliza el costo originalmente en la valuación y posteriormente se refleja cualquier pérdida de valor permanente. De las inversiones temporales se deben de separar las negociables y los dos grupos deben ser divididos en internacionales y nacionales. Las transferencias entre portafolios se registran al costo o mercado el más bajo. La ley prohíbe recibir o pagar intereses sobre los préstamos, por lo que se les llama "comisiones especiales" que se registran bajo el método de acumulación. No hay un procedimiento estandarizado para la clasificación de las deudas con problemas. Los impuestos se calculan sobre los dividendos, así que surgen sumas sustanciales por impuestos diferidos (Hussein, 2002). 


\section{Bangladesh}

Las ganancias y pérdidas por diferencial cambiario requeridas según la IAS 21 no se pueden implementar porque contravienen una disposición de 1994 que obliga a la capitalización de las mismas en cualquier circunstancia (Sacho \& Oberholster, 2008).

\section{Emiratos Árabes Unidos}

Desde 1999 la contabilidad de los bancos debe de prepararse en concordancia con la NICs, pero los intereses de las deudas de dudoso cobro se reconocen sobre la base de efectivo. Además no existe un procedimiento estandarizado para las inversiones; por ejemplo en algunas empresas las inversiones en títulos negociables se registran al valor de mercado y las mantenidas hasta el vencimiento al costo amortizado por el método de línea recta. Otras empresas consideran sus inversiones como de largo plazo y las registran al costo (Hussein, 2002).

\section{España}

España para efectos de la transición aprobó regulaciones un poco diferentes a las establecidas en las NIIF como se muestra en el cuadro siguiente:

CUADRO 1

NIIFS VRS ADAPTACIÓN PARA LA TRANSICIÓN EN ESPAÑA

\begin{tabular}{ccc}
\hline NIIF & España \\
\hline Fecha de transición & $\begin{array}{c}\text { Todas con información } \\
\text { comparativa }\end{array}$ & $\begin{array}{c}\text { Sin información comparativa: 1-1-08 } \\
\text { Con información comparativa: 1-1-07 }\end{array}$ \\
$\begin{array}{c}\text { Valoración de elementos } \\
\text { patrimoniales en la transición }\end{array}$ & $\begin{array}{c}\text { Valorarlos con la nueva } \\
\text { normativa internacional }\end{array}$ & $\begin{array}{c}\text { Valorarlos según la normativa anterior, excepto } \\
\text { los instrumentos financieros que deban valorarse } \\
\text { por su valor razonable o Valorarlos todos con la } \\
\text { nueva normativa }\end{array}$ \\
\hline
\end{tabular}

Fuente: Fitó et al. 2010.

Este país comienza una aplicación uniforme de las normas en todas sus empresas a partir del 2009. Fitó et al., (2010) realizó un estudio entre 33 empresas cotizadas en el mercado continuo español, que adoptaron el 1 de enero de 2007 como fecha de transición y que no son de los sectores financiero $y$ de seguros (por sus normativas particulares). En este análisis se detecta un aumento neto en los reportes de activos, ya que algunos (siguiendo la nueva normativa en comparación a la anterior) deben ser valorados al valor razonable. Este aumento ha compensado los descensos que pudieran causar la eliminación de los intereses diferidos del leasing, los gastos de constitución y la reclasificación en el patrimonio de las acciones propias. Por su parte, el pasivo no corriente aumenta por el impuesto diferido que surge de la aplicación del valor razonable a la valuación de algunos activos.

\section{Estado Unidos}

Una notable ausencia de los países que adoptaron las NIIFs es Estados Unidos. Algunos presumen que sus US GAAP son más robustos $y$ de mayor calidad para proveer de transparencia, consistencia, comparabilidad, relevancia y confiabilidad a la información contable, a pesar de que desde el 2007 SEC aceptó la propuesta de que las compañías extranjeras registradas cuyos estados financieros estuviesen bajo las normas NIIFs no requirieran conciliarlos con US GAAP. Este fue uno de los mayores logros de IASB por establecer un grupo de normas internacionales de contabilidad de alta calidad (Sacho \& Oberholster, 2008).

Por ser este un país tan importante a nivel de mercados financieros es relevante resaltar algunas de las principales diferencias de las US GAAP y las NIIFs, que se pueden ver en el cuadro NIIFs vrs US GAAP, principales DIFERENCIAS. 
CUADRO 2

NIIFS VRS US GAAP, PRINCIPALES DIFERENCIAS

\begin{tabular}{|c|c|c|c|}
\hline & & IASB & US GAAP \\
\hline \multirow{3}{*}{ 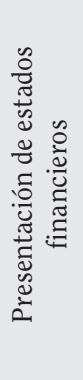 } & $\begin{array}{l}\text { Estado de Resultado } \\
\text { Integral }\end{array}$ & $\begin{array}{l}\text { Los gastos se clasifican por naturaleza } \\
\text { o función }\end{array}$ & Los gastos se clasifican por función \\
\hline & $\begin{array}{l}\text { Estado de Situación } \\
\quad \text { Financiera }\end{array}$ & $\begin{array}{c}\text { No se indica ni el formato ni la } \\
\text { estructura del estado }\end{array}$ & $\begin{array}{c}\text { Activos y pasivos se reportan en orden de } \\
\text { liquidez }\end{array}$ \\
\hline & Flujo de efectivo & $\begin{array}{l}\text { Intereses } y \text { dividendos ganados = } \\
\text { actividades de operación o inversión } \\
\text { Intereses y dividendos pagados } \\
=\text { actividades de operación o } \\
\text { financiamiento }\end{array}$ & $\begin{array}{c}\text { Intereses } y \text { dividendos ganados e intereses } \\
\text { pagados }=\text { actividades de operación } \\
\text { Dividendos pagados = actividad de } \\
\text { financiamiento }\end{array}$ \\
\hline \multirow{9}{*}{ 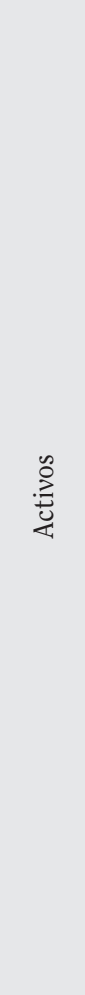 } & \multirow{3}{*}{ Inventario } & Supuestos de flujo: PEPS y Promedio & $\begin{array}{c}\text { Supuestos de flujo: PEPS, UEPS, } \\
\text { Promedio }\end{array}$ \\
\hline & & Incluye costos de diseño & No incluye costos de diseño \\
\hline & & $\begin{array}{l}\text { Costos o valor neto de realización } \\
\text { el menor, valor neto de realización } \\
=\text { precio estimado de venta - costos } \\
\text { estimados de terminación y venta }\end{array}$ & $\begin{array}{c}\text { Costos o mercado el menor, mercado } \\
=\text { costo de reemplazo por compra o } \\
\text { producción }\end{array}$ \\
\hline & $\begin{array}{c}\text { Propiedad, Planta y } \\
\text { equipo }\end{array}$ & $\begin{array}{l}\text { Modelo de costo o modelo de } \\
\text { revaluación }\end{array}$ & Modelo de costo \\
\hline & $\begin{array}{c}\text { Contratos de } \\
\text { construcción de largo } \\
\text { plazo }\end{array}$ & $\begin{array}{l}\text { Método de porcentaje de avance de } \\
\text { preferencia y el de contrato terminado } \\
\text { solamente en casos especiales }\end{array}$ & $\begin{array}{l}\text { Método de porcentaje de avance de } \\
\text { siempre que sea posible y el de cero } \\
\text { ganancia en los demás }\end{array}$ \\
\hline & $\begin{array}{l}\text { Costos de préstamos } \\
\text { para ciertos activos }\end{array}$ & $\begin{array}{l}\text { Da opción de capitalizar o pasar por } \\
\text { gasto }\end{array}$ & Capitalizables siempre \\
\hline & Negocios conjuntos & $\begin{array}{c}\text { Se permite el método de participación y } \\
\text { la consolidación proporcional }\end{array}$ & $\begin{array}{c}\text { Principalmente se usa el método de } \\
\text { participación }\end{array}$ \\
\hline & $\begin{array}{l}\text { Investigación y } \\
\text { desarrollo }\end{array}$ & $\begin{array}{l}\text { Los costos de investigación son gastos } \\
\text { conforme se incurren, pero los de } \\
\text { desarrollo se pueden capitalizar si } \\
\text { cumplen ciertos requisitos }\end{array}$ & $\begin{array}{l}\text { Todos los costos son pasados a gasto } \\
\text { conforme se incurre en ellos }\end{array}$ \\
\hline & Plusvalía & $\begin{array}{l}\text { Registrada al costo, sin amortización, } \\
\text { se evalúa su deterioro cada año }\end{array}$ & $\begin{array}{l}\text { Registrada al costo y amortizada en su } \\
\text { vida útil, menor a } 40 \text { años }\end{array}$ \\
\hline \multirow{3}{*}{$\underset{\substack{\infty \\
:}}{\infty}$} & Pasivos contingentes & $\begin{array}{c}\text { Rango medio de los costos asociados } \\
\text { estimados }\end{array}$ & $\begin{array}{l}\text { Rango más bajo de los costos asociados } \\
\text { estimados }\end{array}$ \\
\hline & & Arrendamientos financieros & $\begin{array}{c}\text { Condiciones para la clasificación: -el } \\
\text { contrato abarca el } 75 \% \text { o más de la vida } \\
\text { útil del activo y -el valor presente de los } \\
\text { pagos es al menos el } 90 \% \text { del valor justo } \\
\text { del activo }\end{array}$ \\
\hline & Pensiones & $\begin{array}{l}\text { Activos del plan - las obligaciones por } \\
\text { los beneficios proyectados más o menos } \\
\text { las ganancias o pérdidas actuariales }\end{array}$ & $\begin{array}{c}\text { Activos del plan - las obligaciones por los } \\
\text { beneficios proyectados }\end{array}$ \\
\hline
\end{tabular}

Fuente: elaboración propia con información de Larson y Lee 2001; Stanko et al. 2010. 


\section{Indonesia}

Indonesia tiene sus propias normas pero el Instituto Indonesio de Contadores usa de base las NIIFs para desarrollarlas (Wilhelm \& Wilhelm, 2010).

\section{Japón}

La Junta de Normas Contables de Japón (ASBJ por sus siglas en inglés) ha trabajado para hacer una convergencia entre los principios contables generalmente aceptados japoneses y las NIIFs. En el 2005, el Comité Europeo Regulador de Valores examinó la comparabilidad de las NIIFs $y$ los ASBJ $y$ concluye que las normas financieras no son equivalentes, lo que significa que las compañías japonesas con capital en los mercados europeos deben de hacer costosos ajustes a sus estados financieros. En 2007 se anuncia un acuerdo para lograr esta convergencia para el 2011 (Kaneko \& Tarca, 2008).

Por ser el segundo mercado de capital más grande del mundo, es prioritario que Japón busque la convergencia con las NIIFs y el camino hacia esta meta comenzó en 1970, cuando a un pequeño número de empresas inscritas en New York Stock Exchange (NYSE) se les permi- tió producir reportes sobre la base de US GAAP (Kaneko \& Tarca, 2008). Luego, desde 1997 a 2002, las normas japonesas son transformadas para hacerlas más compatibles con las US GAAP y las NIIFs en temas como los pagos basados en acciones, las combinaciones de negocios, presentación de estados financieros, ingresos, beneficios a empleado, utilidades por acción, entre otros; (Kaneko \& Tarca, 2008). Sin embargo, en el 2005 aún persistían diferencias significativas en áreas como las fusiones, la amortización de la plusvalía, la contabilización de las subsidiarias, etc. El proceso se puede apreciar en relación al interés minoritario: las normas emitidas para los estados financieros consolidados en 1975, requieren que el interés minoritario se presente dentro de la sección de Pasivos pero en 1997 se cambia la presentación a una sección independiente entre el Pasivo y el Patrimonio (Futamura, 2010).

Kaneko \& Tarca (2008) identifican cuatro importantes áreas para la convergencia de las normas internacionales $y$ las japonesas: el reconocimiento al valor justo, las revelaciones del ingreso integral, la contabilización de los arrendamientos y las combinaciones de negocios. En el siguiente cuadro se muestran las principales diferencias. 
CUADRO 3

NIIFS VRS ASBJ, PRINCIPALES DIFERENCIAS

\begin{tabular}{|c|c|c|c|}
\hline & & IASB & ASBJ \\
\hline 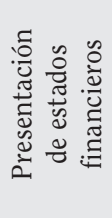 & Estado de Resultado Integral & $\begin{array}{l}\text { Comprende los otros } \\
\text { resultados integrales como los } \\
\text { relacionados con la valuación } \\
\text { de instrumentos financieros, } \\
\text { pensiones y ajustes por } \\
\text { diferenciales cambiarios }\end{array}$ & No existe el término de resultado integral. \\
\hline \multirow{5}{*}{ 竞 } & Propiedad, Planta y equipo & $\begin{array}{l}\text { Modelo de costo o modelo de } \\
\text { revaluación }\end{array}$ & Modelo de costo \\
\hline & Inversiones & $\begin{array}{l}\text { Las inversiones negociables se } \\
\text { valúan al valor de mercado y la } \\
\text { ganancia o pérdida resultante } \\
\text { se lleva al estado de resultado }\end{array}$ & $\begin{array}{l}\text { La comunidad japonesa tiene una tradición } \\
\text { de mantener inversiones cruzadas de } \\
\text { acciones entre empresas que se presentan } \\
\text { como inversiones en otros valores }\end{array}$ \\
\hline & Combinaciones de negocios & $\begin{array}{l}\text { Requiere el método de } \\
\text { compra, la plusvalía se revisa } \\
\text { anualmente para analizar su } \\
\text { deterioro }\end{array}$ & $\begin{array}{l}\text { Algunas empresas todavía utilizan el método } \\
\text { de "pooling" }\end{array}$ \\
\hline & Plusvalía & $\begin{array}{l}\text { Registrada al costo, sin } \\
\text { amortización, se evalúa su } \\
\text { deterioro cada año }\end{array}$ & $\begin{array}{l}\text { Se amortiza la plusvalía en un periodo } \\
\text { máximo de } 20 \text { años }\end{array}$ \\
\hline & Arrendamientos financieros & $\begin{array}{l}\text { Bajo ciertas condiciones se } \\
\text { capitalizan }\end{array}$ & $\begin{array}{l}\text { Los arrendamientos financieros que no } \\
\text { transfieren la propiedad se registran como } \\
\text { arrendamientos operativos, en los estados. La } \\
\text { información adicional se revela en las notas }\end{array}$ \\
\hline
\end{tabular}

Fuente: elaboración propia con información de Kaneko \& Tarca, 2008.

\section{Luxemburgo}

Este país permite a sus declarantes extranjeros usar NIIFs, UK GAAP, US GAAP o los principios contables de su propio país si se reconcilian con NIIFs.

\section{México}

En México tienen sus propios principios contables generalmente aceptados pero las reglas mexicanas requieren que se sigan las NIIFs en ausencia de alguna guía en los PCGA Mexicanos (Wilhelm \& Wilhelm, 2010).

\section{Omán}

La plusvalía se reconoce como un activo intangible y se amortiza en un máximo de 5 años, a menos que una vida útil mayor pueda ser justificada. Las inversiones se registran al costo o el mercado el menor y los bonos del tesoro a su valor facial. (Hussein, 2002).

\section{Qatar}

Las inversiones se registran al costo, con una provisión para cualquier disminución del valor en el portafolio (Hussein, 2002).

\section{Suráfrica}

En este país se permite aplicar tanto los principios contables generalmente aceptados nacionales como las NIIFs desde 1993 lo que ha provocado una convergencia de algunos principios (Wilhelm \& Wilhelm, 2010). 


\section{Tailandia}

Adopta las normas pero omite secciones como la IAS 39 y 14 y la plusvalía según la NIIF 3, porque se considera que podría tener consecuencias negativas en las compañía ubicadas en mercados emergentes (Sacho \& Oberholster, 2008).

\section{Los nuevos retos de IASB}

Aún hay algunos retos que la IASB debe afrontar en el futuro (Zeff, 2012):

- $\quad$ lograr que Estados Unidos, China y Japón adopten las normas;

- equilibrar la diversidad de retroalimentación recibida de sus nuevos integrantes como América Latina y Oceanía;

- $\quad$ inspirar en los reguladores de los mercados de valores el control $y$ vigilancia para que las compañías inscritas cumplan con las NIIF y ser más proactivos cuando notan deficiencias en los reportes financieros;

- definir la forma en que debe de declararse el cumplimiento de las IFRS de forma total en los estados auditados, sin los calificativos de "adoptados por la Unión Europea" sino "IFRS emitidos por IASB";

- $\quad$ solucionar la forma en que son aplicadas las normas sobre las diferentes formas de negocios alrededor del mundo sin dejar de promover la comparabilidad,

- dar igual participación y acceso a las partes interesadas.

En la opinión de autores como Sacho \& Oberholster (2008), IASB aún es una institución vulnerable ante factores externos como:

- La influencia de los grupos de presión y proveedores de fondos

- $\quad$ La influencia de Estados Unidos sobre la emisión de las normas internacionales de contabilidad.

- Los escándalos como un resultado de la mala aplicación de los principios contables fundamentales
- Las diferentes interpretaciones y aplicaciones de las normas de contabilidad debido a las diferencias culturales

Por ejemplo, durante la crisis económica iniciada en el 2008, tanto la IASB como la FASB se vieron ante el dilema de proveer información transparente a los inversionistas en los mercados de capitales o ceder a la presión de los bancos de proyectar estabilidad financiera, lo que puso en entredicho su capacidad de mantenerse firmes en los principios contables (Zeff, 2012).

Cuando la Unión Europea nombra el European Financial Reporting Advisory Group (EFRAC) en el 2001, que veta las NIIF antes de su adopción en Europa, mina el intento de IASB de crear una normativa contable global (Richardson y Eberlein, 2011).

Las normas han tratado de abarcar las transacciones más rutinarias pero siempre existen vacíos en las regulaciones que deben ser aclarados, por ejemplo en la NIC 2 no se menciona la forma de calcular la capacidad normal $y$ hay cierta subjetividad al definir el valor neto realizable (Morillo, 2010).

\section{CONCLUSIONES}

La información financiera es base para la toma de decisiones en los negocios y por ello los países se preocuparon en el pasado por establecer normas que dictaran la forma de preparar y divulgar esta información. Cuando los negocios se circunscribían al ámbito nacional, no había problemas con la variabilidad de estos estándares internacionalmente, pero conforme se hacen transacciones que cruzan las fronteras de los países, los problemas de comparabilidad comienzan a darse. De allí que los principales interesados en crear normas internacionalmente aceptadas sean las Bolsas de Valores, que aceptan capitales de diferentes nacionalidades y deben justificar su riesgo a través de los estados financieros.

La IASC nace como un primer intento de unificar estas prácticas contables a través de sus NICs y más tarde la IASB lo hace incorporando a estas normas internacionales las NIIFs, con la intención de tener un nivel mínimo de 
calidad en la información financiera. Así se logran emitir 41 NICs y 13 NIIFs hasta el 2013, cuya aplicación se ha extendido alrededor del mundo. También en el 2009 el IASB emite las normas contables para las empresas que no tienen obligación pública de rendir cuentas (definidas como PYMES para estos efectos) con el fin de simplificar su uso para este sector empresarial.

Ya para el 2005, 8.000 de las empresas inscritas en las bolsas de valores europeas presentaron sus estados obedeciendo las NIIFs y países que no las han adoptado todavía, como Japón y Estados Unidos, flexibilizaron un poco sus reglas para que empresas extranjeras inscritas en sus propias bolsas de valores, pudieran presentar la información financiera sobre la base de las NIIFs.

El impacto de la aplicación de las normas ha sido medido en algunos países, donde se ha notado:

a) aumento significativo de partidas de activo,

b) reducción de provisiones $y$

c) modificaciones en el ingreso neto debido a las nuevas regulaciones que:

a. eliminan la amortización de la plusvalía,

b. requieren la medición de algunos instrumentos financieros al valor de mercado,

c. exigen el reconocimiento de algunos costos de desarrollo (en procesos de Investigación $y$ desarrollo), $y$

d. exigen el reconocimiento de impuestos diferidos.

Algunos países han implementado estas normas tal $y$ como fueron emitidas, pero otros han hecho ajustes en su aplicación, aunque se encuentren en procesos de convergencia total. Esto ha dificultado que cumplan con su objetivo de producir información financiera transparente y comparable. Otros países no las han adoptado, como Japón y Estados Unidos, pero están en constante negociación para llegar a una redacción de estándares que se ajuste a las necesidades de información financiera de los inversionistas.
Aún quedan vacíos en las normas que permiten interpretaciones muy diversas, también se deben de eliminar los tratamientos alternativos que dificultan la comparabilidad. Otros de los retos que debe de asumir la IASB es hacer que las normas se apliquen de forma estricta, sin variaciones entre países y no ceder a los grupos de presión y proveedores de fondos que pretenden influir en su redacción para beneficio individual.

En Costa Rica, está por investigarse si las normas están siendo aplicadas de manera uniforme e integral en los grupos empresariales que presentan información al público y cuál ha sido el impacto en las cifras de sus estados financieros. También es importante determinar las dificultades que hayan encontrados las Pymes para aplicar las normas que les son específicas.

\section{REFERENCIAS}

Callao, S.; Larne, J. \& Laínez, J. (2007) "Adoption of IFRS in Spain: Effect on the comparability and relevance of financial reporting". Journal of International Accounting, Auditing and Taxation. v.16, p. $148-178$.

Canfield, C. (1999). FASB v. IASC: Are the structure and standard setting process at the IASC adequate for the Securities and Exchange Commission to accept international accounting standards for cross-border offerings. North-western Journal of International Law \& Business, 20(1), 125-144.

Chasipanta G., R. (2012) Estudio del impacto en la aplicación de las normas internacionales de información financiera (NIIF para PYMES) en la compañía Guarderas S. A. Tesis previa a la obtención del título de Ingeniera en Contabilidad y Auditoría, Universidad Central del Ecuador.

Cordazzo, M. (2008). The impact of ias/ifrs on accounting practices: evidences from Italian listed companies. Documento presentado en XXXI Congress of European Accounting Association, Rotterdam. 
Fitó, M. À., Gómez, F., \& Moya, S. (2010). Efectos del nuevo PGC en los estados financieros: El problema de la comparabilidad de los datos/The effects on comparability of the new local accounting standards in Spain. Universia Business Review, (28), 136-149.

Futamura, M. (2010). The introduction of accounting principles for consolidated financial statements in Japan: focus on minority interest and other related accounting treatments. Journal of International Business Research, Volume 9, Special Issue 1, 1-23.

Hung; M. \& Subramanyam, K. (2004) "Financial Statement Effects of Adopting International Accounting Standards: the Case of Germany". Review of Accounting Studies, Forthcoming.

Hussain, M.; Islam, Mazhar M; Gunasekaran, A; Maskooki, Kooros (2002). Accounting standards and practices of financial institutions in GCC countries. Managerial Auditing Journal; 17, 7.

Kaneko, Sei-Ichi \& Tarca, Ann. (2008). Converging to International Accounting Standards: Views from Japan. Australian Accounting Review; Sep.; 18, 3; pg. 209.

Jara, L. \& Contreras H. (2010). Adopción de IFRS en empresas chilenas. Énfasis en los efectos cuantitativos sobre la información financiera. CAPIC REVIEW Vol. 8 ISSN 0718-4654.
Larson, R. K., \& Linda, L. L. (2001). Coming to terms with international accounting standards. The Internal Auditor, 58(1), 42-47.

Morillo M., M. La contabilidad de costos y la adopción en Venezuela de las normas internacionales. Actualidad Contable FACES Año $13 \mathrm{~N}^{\circ}$ 21, Julio- Diciembre 2010. Mérida. Venezuela (90-115)

Richardson, A. \& Eberlein, B. (2011). Legitimating Transnational StandardSetting: The Case of the International Accounting Standards Board. Journal of Business Ethics 98:217-245.

Sacho, Z. \& Oberholster, J. (2008) Factors impacting on the future of the IASB. Meditari Accountancy Research Vol. 16 No. 1: 117-137

Stanko, B. B., Zeller, T. L. (2010). The arrival of a new GAAP: International financial reporting standards. Journal of Business \& Economics Research, 8(10), 27-37.

Wilhelm, P. \& J. Wilhelm (2010). An analysis of corruption, taxation and the IASB: the effect on global competitiveness. ACR Vol.18 (1\&2), 140-163.

Zeff, S. A. (2012). The evolution of the IASC into the IASB, and the challenges it faces. The Accounting Review, 87(3), 807-837. 